Proceeding Series of the Brazilian Society of Computational and Applied Mathematics

\title{
Análise e comparação preliminar de fluxos numéricos usuais em sistemas de magneto-hidrodinâmica
}

\section{Daniele Pereira da Silva ${ }^{1}$}

Universidade do Estado do Rio de Janeiro, UERJ, RJ

Instituto Politécnico, IPRJ, Nova Friburgo, RJ

Margarete Oliveira Domingues ${ }^{2}$

Instituto Nacional de Pesquisas Espaciais, INPE, São José dos Campos, SP

Laboratório Associado de Computação e Matemática Aplicada, LAC/CTE

João Flávio Vieira de Vasconcellos ${ }^{3}$

Universidade do Estado do Rio de Janeiro, UERJ, RJ

Instituto Politécnico, IPRJ, Nova Friburgo, RJ

Edgard Freitas Diniz Evangelista ${ }^{4}$

Bolsista do CNPq-Brasil

Instituto Nacional de Pesquisas Espaciais, INPE, São José dos Campos, SP

Resumo.O presente trabalho propõe revisar, analisar e discutir as metodologias da técnica de volumes finitos utilizadas para o cálculo do fluxo numérico em modelos magneto-hidrodinâmicos (MHD). O cálculo do fluxo nas regiões descontínuas destas equações é uma tarefa de grande custo computacional, portanto apresenta-se os primeiros resultados da comparação da eficiência numérica computacional de três diferentes esquemas existentes na literatura: HLL, HLLD e HLLEM.

Palavras-chave. Magneto-hidrodinâmica, Fluxos numéricos, Volumes finitos.

\section{Introdução}

Nesta pesquisa, tem-se interesse em contribuir com a melhoria da modelagem do plasma espacial estudando técnicas numéricas para resolver as equações de magnetohidrodinâmica. A magneto-hidrodinâmica (MHD) é uma área do conhecimento cujo ob-

\footnotetext{
${ }^{1}$ danielepereiradasilva@yahoo.com.br

${ }^{2}$ margarete.domingues@inpe.com.br

3 jflavio@iprj.uerj.br

${ }^{4}$ edgard.evangelista@inpe.br
} 
jetivo de estudo é o movimento de fluidos condutores submetidos à influência de campos eletromagnéticos. O modelo magneto-hidrodinâmico é uma extensão das equações de dinâmica dos fluidos, com a inclusão dos efeitos da força eletromagnética [2]. Uma das técnicas utilizadas para o estudo dessa modelagem é o método de volumes finitos. Nessa técnica faz-se necessário adicionar correções ao modelo MHD de forma a manter a condição de divergência nula do vetor campo magnético $[1,8]$. Há várias opções para isto. Neste estudo utiliza-se o modelo Extensão Generalizada dos Multiplicadores de Lagrange (EGLM-MHD) proposto em [1] que introduz termos fontes a certas equações do modelo para manter controlada a divergência de $\mathbf{B}$ e assim evitar que artifícios numéricos contaminem a solução física esperada.

Ao utilizar o método dos volumes finitos para simulação destas equações é necessário calcular o fluxo numérico. Nos últimos anos uma grande variedade de Riemann Solvers têm sido desenvolvidos para melhorar a eficiência das simulações [9]. Em [10] a comparação dos fluxos em um sistema MHD é testada utilizando um método Galerkin descontínuo. Neste trabalho são implementados os fluxos numéricos Harteen Lax Van Leer (HLL) [4], Harteen Lax Van Leer Descontínuo (HLLD) [7] e Harteen Lax Van Leer Melhorado (HLLEM) [9] utilizando o método dos volumes finitos [6] em uma malha estruturada. Os resultados são analisados e comparados.

\section{Modelo MHD}

O modelo MHD ideal é derivado das equações de Euler para dinâmica dos gases acoplado com as equações de Maxwell, onde utiliza-se uma equação evolutiva adicional para o campo magnético. Neste trabalho utiliza-se a formulação do modelo MHD ideal denominada Extensão Generalizada dos Multiplicadores de Lagrange (EGLM). Esta formulação é apresentada em [1]. As equações deste modelo EGLM-MHD ideal representam a conservação da massa, momento, energia e campo magnético, respectivamente:

$$
\begin{aligned}
\frac{\partial \rho}{\partial t}+\nabla \cdot(\rho \mathbf{u}) & =0 \\
\frac{\partial \rho \mathbf{u}}{\partial t}+\nabla \cdot\left[\rho \mathbf{u u}+\left(p+\frac{\mathbf{B} \cdot \mathbf{B}}{2}\right) \mathbf{I}-\mathbf{B B}\right] & =-\mathbf{B}(\nabla \cdot \mathbf{B}) \\
\frac{\partial \mathbf{B}}{\partial t}+\nabla \cdot(\mathbf{u B}-\mathbf{B u}+\psi \mathbf{I}) & =0 \\
\frac{\partial E}{\partial t}+\nabla \cdot\left[\left(E+p+\frac{\mathbf{B} \cdot \mathbf{B}}{2}\right) \mathbf{u}-(\mathbf{u} \cdot \mathbf{B}) \mathbf{B}\right] & =-\mathbf{B} \cdot(\nabla \psi) \\
\frac{\partial \psi}{\partial t}+c_{h}^{2} \nabla \cdot \mathbf{B} & =-\frac{c_{h}^{2}}{c_{p}^{2}} \psi
\end{aligned}
$$

em que $\rho$ representa a densidade, $p$ é a pressão, $\mathbf{u}=\left(u_{x}, u_{y}, u_{z}\right)$ é o vetor velocidade , $\mathbf{B}=\left(B_{x}, B_{y}, B_{z}\right)$ é o vetor campo magnético, $\mathbf{I}$ é o tensor identidade de segunda ordem e $\psi$ é a função potencial. A energia é dada pela lei 


$$
E=\frac{p}{\gamma-1}+\rho \frac{\mathbf{u} \cdot \mathbf{u}}{2}+\frac{\mathbf{B} \cdot \mathbf{B}}{2}
$$

e $\gamma$ é uma constante adiabática $(\gamma>1)$. O campo magnético $\mathbf{B}$ é livre de divergência, i.e. $\nabla \cdot \mathbf{B}=0$.

Geralmente a incompressibilidade do campo magnético não é preservada na solução numérica, e assim, pode-se encontrar uma solução não-física e os cálculos tornarem-se instáveis. Dessa forma, deve-se implementar correções de divergência para manter esta restrição. Neste trabalho, utiliza-se a correção parabólica-hiperbólica proposta em [1].

Associadas ao modelo MHD existem três velocidades de propagação do som: rápida (do termo em inglês fast, expressa por $c_{f}$ ), Álfven (expressa por $c_{a}$ ) e lenta (do termo em inglês slow, expressa por $c_{s}$ ) [8]. Estas velocidades estão relacionadas com os autovalores e autovetores do sistema e são descritas pelas equações:

$$
\begin{gathered}
c_{f, s}=\sqrt{\frac{1}{2}\left(a^{2}+b^{2} \pm \sqrt{\left.\left(a^{2}+b^{2}\right)^{2}-4 a^{2} b_{k}^{2}\right)}\right.} \\
c_{a}=\left|b_{k}\right|
\end{gathered}
$$

em que $a=\sqrt{\frac{\gamma p}{\rho}}, b=\sqrt{\frac{|B|^{2}}{\rho}}$

\section{O método dos volumes finitos}

Para realizar a simulação numérica das equações é necessário a utilização de métodos numéricos. Neste trabalho propõem-se o método dos volumes finitos, que considera a forma integral da equação conservativa. O domínio do problema é particionado em células de grade e utiliza-se o esquema:

$$
\frac{\partial \mathbf{U}}{\partial t}+\nabla \cdot \mathbf{F}(\mathbf{U})=0
$$

em que $\mathbf{U}=\left(\rho, p, \psi, v_{x}, v_{y}, v_{z}, B_{x}, B_{y}, B_{z}\right)$ é o vetor de variáveis e $\mathbf{F}(\mathbf{U})$ é o fluxo físico.

Para o cálculo de $\mathbf{F}(\mathbf{U})$ deve-se utilizar aproximações numéricas, chamadas fluxos numéricos ou Riemann Solvers. Neste trabalho, utiliza-se as aproximações HLL [4], HLLD [7] e HLLEM [9], conforme apresentados em mais detalhes a seguir.

\subsection{Método Harten Lax Van Leer - HLL}

O método HLL [4] utiliza uma configuração para a solução que consiste de duas ondas que separam três estados constantes. O fluxo para o estado intemediário é representado por $F_{H L L}=\frac{S_{R} F_{L}-S_{L} F_{R}+S_{R} S_{L}\left(U_{R}-U_{L}\right)}{S_{R}-S_{L}} \operatorname{com} S_{L}=\min \left(u_{L}-c_{L}, u_{R}-c_{R}\right), S_{R}=\max \left(u_{L}+\right.$ $\left.c_{L}, u_{R}+c_{R}\right)$ em que $\mathrm{L}$ e $\mathrm{R}$ são as células da direita e esquerda, respectivamente. Os valores $u_{L}$ e $u_{R}$ são as velocidades do plasma e $c_{L}$ e $c_{R}$ as velocidades magnetoacústicas. Os valores $F_{L}$ e $F_{R}$ são os vetores do fluxo e $U_{L}$ e $U_{R}$ os vetores das variáveis. 


\subsection{Método Harten Lax Van Leer Discontinuous - HLLD}

O Riemann solver HLLD [7] pode ser visto como uma forma de evolução das inconsistências no que diz respeito às condições de salto encontradas no método HLL. Isto porque o HLLD gera uma configuração de cinco ondas que produzem quatro estados intermediários. Esta configuração gera uma solução aproximada não linear, onde se obtém uma solução mais precisa do problema. Uma forma mais detalhada do método pode ser encontrada em [3].

\subsection{Método Harten Lax Van Leer Modified - HLLEM}

O esquema HLLEM [9] foi desenvolvido como uma viabilidade de melhoria do esquema HLL. O método consiste em introduzir um termo anti-difusão na onda de entropia. Para o cálculo do esquema utiliza-se a média aritmética das variáveis de estado. A função de fluxo numérico para o método é dada por $F_{H L L E M}=F_{H L L}+\phi_{1} a\left(U_{L}, U R\right)$ em que $a\left(U_{L}, U_{R}\right)=$ $\frac{S_{R} S_{L}}{S_{R}-S_{L}} \sum_{i=2}^{6} \delta_{i} \alpha_{i} r_{i}$ é o termo anti-difusão. Os coeficientes $\delta_{i}$ e $\phi_{1}$ estão relacionados às velocidades magnetoacústicas e $\alpha_{i} r_{i}$ estão relacionados com os autovetores do sistema.

Este método possui um custo computacional alto pois necessita do cálculo dos autovetores do sistema, o que não é uma tarefa simples. O ajuste dos parâmetros das variáveis também deve ser feito com cuidado. Detalhes da implementação podem ser encontrados em $[9]$.

\section{Resultados}

O modelo EGLM-MHD 2D em volumes finitos utilizado para a implementação do fluxo HLLEM realizada neste trabalho foi desenvolvido por [3].

Para este estudo utilizam-se as condições iniciais de um problema tipo Riemman 1D apresentadas na Tabela 1 e discutidas em [5]. Considera-se também o domínio $[-0.5 \times 0.5]$. Neste caso pode-se obter uma solução exata para comparação ${ }^{1}$.

Tabela 1: Condição inicial Riemann 1D.

\begin{tabular}{|c|c|c|c|c|c|c|c|c|}
\hline & $\rho$ & $p$ & $v_{x}$ & $v_{y}$ & $v_{z}$ & $B_{x}$ & $B_{y}$ & $B_{z}$ \\
\hline$x \leq 0$ & 1.08 & 0.95 & 1.20 & 0.01 & 0.50 & $2.00 / \sqrt{4 \pi}$ & $3.60 / \sqrt{4 \pi}$ & $2.00 / \sqrt{2 \pi}$ \\
$x>0$ & 1.00 & 1.00 & 0.00 & 0.00 & 0.00 & $2.00 / \sqrt{4 \pi}$ & $4.00 / \sqrt{4 \pi}$ & $2.00 / \sqrt{2 \pi}$ \\
\hline
\end{tabular}

Para as simulações são definidos ainda os seguintes parâmetros: $C F L=0.3, \gamma=\frac{5}{3}$, tempo final $t=0.1$ e uma malha regular $500 \times 500$ pontos. As variáveis da solução numérica do modelo EGLM-MHD com os três fluxos numéricos são comparadas com sua solução exata. Nas Figuras (1) a (3) têm-se a solução exata (em vermelho), solução numérica com

\footnotetext{
${ }^{1}$ https://web.mathcces.rwth-aachen.de/mhdsolver/
} 
o método HLL (em verde), solução numérica com o método HLLD (em azul) e solução numérica com o método HLLEM (em magenta).
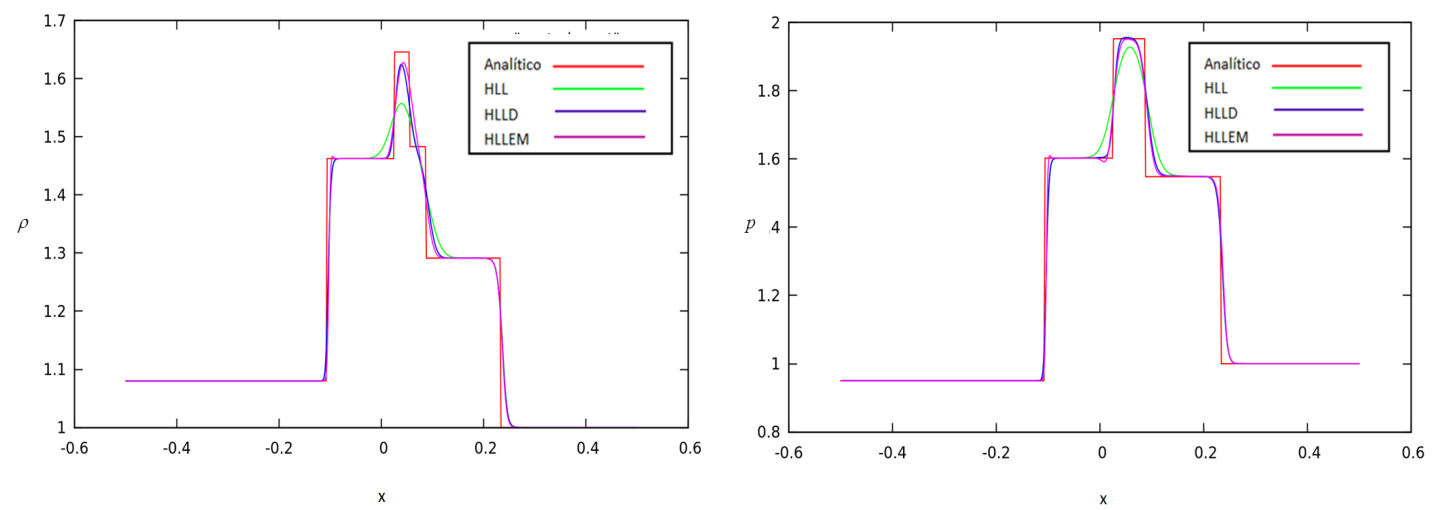

Figura 1: Variáveis densidade $(\rho)$ e pressão $(\mathrm{p})$
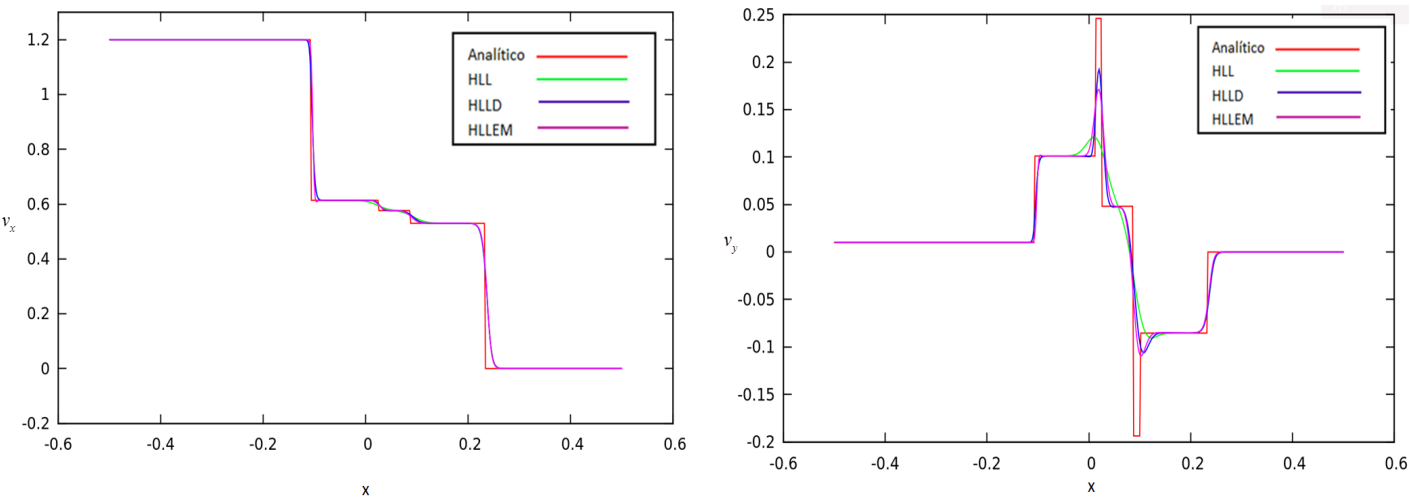

Figura 2: Variáveis $v_{x}$ e $v_{y}$
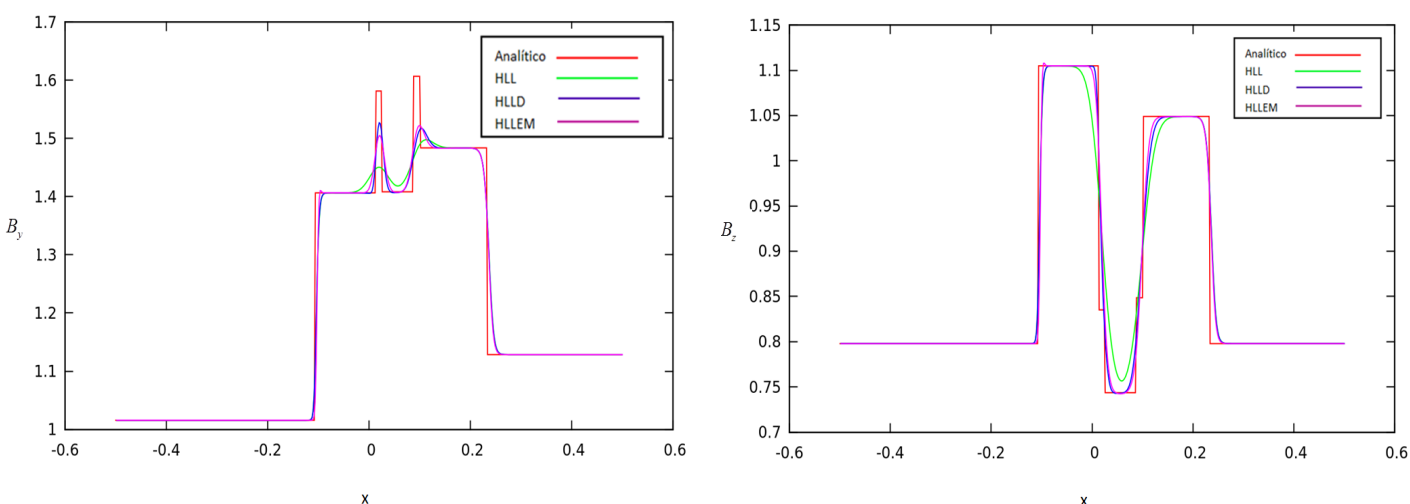

Figura 3: Variáveis $B_{y}$ e $B_{z}$ 


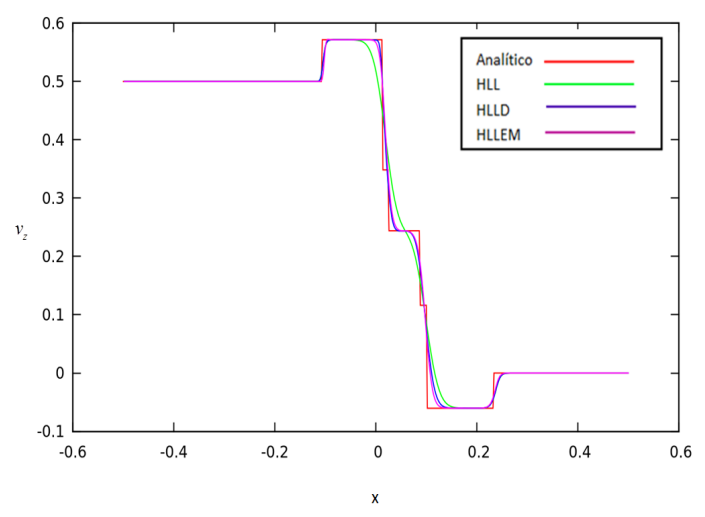

Figura 4: Variável $v_{z}$

\subsection{Análise de erros}

A comparação dos esquemas é feita com uma análise de erros utilizando a solução exata e a solução numérica. Para o cálculo do erro utiliza-se a expressão:

$$
\text { Erro }=\sqrt{\frac{\sum_{i=1}^{N_{x} N_{y}}(\text { Numérico-Analítico })^{2}}{\sum_{i=1}^{N_{x} N_{y}}(\text { Analítico })^{2}}}
$$

em que $N_{x}$ é a quantidade de células na direção x, $N_{y}$ a quantidade de células na direção y, Numérico e Analítico são as soluções numérica e de referência, respectivamente. A Tabela 2 apresenta os resultados em diferentes tamanhos de malha: $200 \times 200$ e $500 \times 500$.

Tabela 2: Análise de erros

\begin{tabular}{|c|c|c|c|c|c|c|c|c|}
\hline Método & Malha & $\rho$ & $\mathrm{p}$ & $v_{x}$ & $v_{y}$ & $v_{z}$ & $B_{y}$ & $B_{z}$ \\
\hline HLL & $200 \times 200$ & 0.03305 & 0.05314 & 0.05235 & 0.10156 & 0.06242 & 0.03248 & 0.04441 \\
\hline HLL & $500 \times 500$ & 0.02399 & 0.03845 & 0.03528 & 0.0841 & 0.04678 & 0.02447 & 0.03126 \\
\hline HLLD & $200 \times 200$ & 0.02756 & 0.04522 & 0.05151 & 0.08563 & 0.04080 & 0.02981 & 0.03237 \\
\hline HLLD & $500 \times 500$ & 0.01952 & 0.03244 & 0.03436 & 0.06461 & 0.02914 & 0.02113 & 0.0230 \\
\hline HLLEM & $200 \times 200$ & 0.02491 & 0.04219 & 0.04827 & 0.08438 & 0.04007 & 0.02901 & 0.03206 \\
\hline HLLEM & $500 \times 500$ & 0.02025 & 0.03136 & 0.03507 & 0.06620 & 0.02923 & 0.02235 & 0.02415 \\
\hline
\end{tabular}

\section{Considerações finais}

A Tabela 2 mostra que com o refinamento da malha a solução é melhorada. O método menos acurado para todas as variáveis é o HLL. Este método possui uma implementação 
computacional mais facilitada, se comparado aos outros dois esquemas. Os métodos HLLD e HLLEM possuem valores semelhantes na avaliação de erros e mais próximos à solução analítica, tornando-os os mais indicados para o cálculo do fluxo em regiões de descontinuidades. Estes resultados podem ser utilizados para pesquisas em resolução de sistemas de magneto-hidrodinâmica em regiões de descontinuidade, em especial para o plasma espacial.

\section{Agradecimentos}

Os autores agradecem à CAPES e ao CNPq, processo número 158967/2014-3 pelo apoio financeiro.

\section{Referências}

[1] A. Dedner, F. Kemm,D. Kroner, C. Munz, T. Schnitzer, and M. Wesenberg, Hyperbolic divergence cleaning for the mhd equations, Journal of Computational Physics, 175(2): 645-673, (2002).

[2] J. Bittencourt, Fundamentals of plasma physics, Springer-Verlag New York, (2004).

[3] A. K. F. Gomes, M. O. Domingues, K.Schneider, O. Mendes and R. Deiterding. An adaptative multiresolution method for ideal magnetohydrodynamics using divergence cleaning with parabolic-hyperbolic correction, Applied Numerical Mathematics, (2015).

[4] A. Harten, High resolution schemes for hyperbolic conservation laws, Journal of computational physics, 49(3):357-393, (1983).

[5] S. Li, An HLLC Riemann Solver for magneto-hydrodynamics, Journal of computational Physics, 203:344-357, (2005).

[6] C.R. Maliska, Transferência de calor e mecânica dos fluidos computacional, LTC, (2005).

[7] T. Miyoshi, T. and K. Kusano, A multi-state hll approximate riemann solver for ideal magneto-hydrodynamics. Journal of Computational Physics, 208(1):315-344, (2005).

[8] K. G. Powell, P. L. Roe, T. J. Linde, T. I. Gombosi and D. L. D. Zeeuw, A SolutionAdaptative Upwind Scheme for Ideal Magnetohydrodynamics, Journal of Computational Physics, 154:284-309, (1999).

[9] M. Wesenberg, Efficient Finite-Volume Schemes for Magnetohydrodynamic Simulations in Solar Physics, PhD thesis, Albert-Ludwigs-Universitat Freiburg im Breisgau, (2003).

[10] V. Wheatley, H. Kumar and P. Hugenot, On the role of Riemann solvers in discontinuous Galerkin methods for magnetohydrodynamics, Journal of computational Phiysics, 239(3), 660-680, (2010). 